\title{
Evaluation of Training Student Reviewers for the Purpose of Enhancing Writing Quality in ESL Writing Classes
}

\author{
Jamal A. Khlifat, MA, MSLS \\ Department of Linguistics \\ University of Colorado, Boulder
}

\begin{abstract}
This action research project investigates the effectiveness of the peer-review process in ESL writing classes. The research adopts the peer-review process by using different pedagogical techniques during this process to evaluate the effectiveness of this process in general and each pedagogical approach used to achieve it in particular, more specifically the peer-review letter adopted by some rhetoricians at CU, Boulder. The participants in this case study are four students - three from China and one from Saudi Arabia-in WRTG 1150, a class targets first-year writing and rhetoric. The students have participated in a series of peer-review processes, providing feedback to their peers on both the global level of the writing process and the formal level. Data has been collected from students' responses to some questions given to them in forms of handouts during the peer-review process, students' revised work, interviews, and the peer-review letter mentioned above. Although helpful, the results of this study indicate that there are factors such as the learner's age, academic level, bias, pride, and cultural background that seem to play a role in the amount of feedback given and incorporated. The results also show that while the peer-review has seemed helpful on the global level of the writing process, it has not done so on the formal level of the writing process. The results also reveal, to a certain extent, that there is no a-winner-takes-all pedagogical tool can be adopted to carry out this process. The teacher should take into consideration his/her students' ages, academic levels, and cultural backgrounds.
\end{abstract}

\section{INTRODUCTION}

\subsection{Overview}

The title of this action research project reveals the main knot that it seeks to untangle. This action research project, which will be part of a large-scale one I am planning to conduct over 2016's summer, speaks to some of the teaching practices and theories that have addressed the notion of peerreview in writing classes.

\subsection{Statement of the Problem/ Focus of Inquiry}

An article written by Rahimi in the field of composition and rhetoric claiming that training student reviewers for the purpose of providing writing feedback to their peers not only helps reviewees strengthen their writing/rhetoric skills but also helps reviewers enhance their own rhetoric skills. His article and recommendations have, to a certain extent, contradicted my past experience of observing students provide writing feedback to one another.

Based on my experience in teaching writing classes, other factors such as the cultural background, age, language (the linguistic background), and gender category of the participants could become major players in this field, which might lead to channel the impact of this approach into the opposite of what the teacher aims for.

This research project aims first and foremost at capturing a good picture of how the peer-review process in a writing class might be helpful - especially when participants have come from educational and cultural backgrounds other than English. The study also tries to shed light on how introducing and using the peer-review letter, a pedagogical technique used by Dr. Andrea Feldman and Jennifer Campbell in writing classes at CU, Boulder, might be helpful in motivating students to provide better instructive feedback to their peers on both the global level of the writing process (cohesion, development, and organization) and the formal level of the writing process (grammar, structure, and writing conventions). 


\subsection{Inquiry Questions}

My overarching questions driving this action research project include the followings:

1. What impact, if any, does the peer-review process have on the reviewer's and reviewee's writing skills in terms of enhancing their global and/or formal writing skills?

2. What roles, if any, can the learner's characteristics such age, pride, biases, culture, and language play in "training student reviewers"?

3. How does likely the use of the peer-review letter influence the peer-review process in terms of the amount of feedback students incorporate in their revised work?

\section{LITERATURE REVIEW}

\subsection{General Scaffolding Techniques and the "Zone of Proximal Development"}

Since we are discussing the impact that trained writing reviewers can leave on their peers (reviewees), I feel an urgent need to touch upon a very important term and technique that has proved itself fruitful in such pedagogical environments, serving as the base for the approach of training student reviewers, viz., scaffolding. Scaffolding in general can be defined as any kind of potential assistance that can be given to a learner in order to accomplish what would have been too difficult for him/her to accomplish on his/her own. This potential assistance is only achieved by making the input of the learning process comprehensible to the learner. Scaffolding has been seen as a very effective strategy and technique to make input comprehensible to second language learners. It definitely goes beyond making the integral subject content comprehensible, contributing to faster and more effective acquisition of the input.

Based on the literature that has examined this technique and based on my own experience with using it to help ESL learners increase their lexical repository (lexicon), I have undoubtedly come to believe that although it has its own drawbacks on the lexical and semantic levels, scaffolding should be praised to walk the learner out of his/her independent zone of learning to what Vygotsky refers to as the "Zone of Proximal Development" or "ZPD" (Shoebottom). "Zone of Proximal Development" is that potential progress that a student could reach with the help of a teacher or a more knowledgeable peer. In other words, it is that notional gap between the learner's current level of development when adopting learning and problem-solving strategies independently and that level of potential development when an adult teacher or a more capable or knowledgeable peer contributes to the learning and problem-solving strategies by guiding and helping the learner. The instructor's guidance and help can be best envisioned as a beam of light that tends to help the learner walk out of his/her current zone of development. This is due to the fact that when learners are in their own zone of development, meaning learning the input independently, the input may not be fully comprehensible, and a more knowledgeable peer's or an instructor's guidance is needed to achieve this goal. When such input is made comprehensible, a useful and helpful milieu conducive tolearninginput is made available.

In our approach, the teacher, as a more knowledgeable individual, must take the lead and train his/her student reviewers to help them acquire the necessary skills in writing. By doing so, the teacher helps his/her student trainees reach their "Zone of Proximal Development" in regard to two levels of skills. The first level targets the global issues of the writing process, which are sentence/paragraph cohesion, idea development, and organization. The second level targets the formal issues of the writing process, which are sentence/paragraph structure, grammar, punctuation, and stylistic norms. This method is actually the core of some studies that have targeted "training student reviewers" in this regard such as Rahimi, 2013; Berg, 1999, Wertsch; 1979; and a few others. When these student trainees master these skills and reach their "Zone of Proximal Development" in these two levels, they will act as more knowledgeable peers to their reviewees to help them reach their Zone of Proximal Development. This is why the entire approach is scaffolding-based.

\subsection{Three Factors might affect Scaffolding and the Notion of "Zone of Proximal Developments, Thus Affecting the Peer-Review Process}

The first factor that might impact the ways in which diverse groups of students can be scaffolded is that of face-saving. According to Monfret, this factor emanates from a proverb that some cultures believe in, i.e., "Spilled water is hard to regain" (2). I have taught international students for few years, and I have sensed a sort of pattern in the cultural and educational milieus where I have taught. The 
concept of "face-saving" can be the very nightmare that keeps scaffolding up at night, leading to undermine all of its efforts. This concept tends to become very active if the reviewers and their reviewees have come from different cultural backgrounds. Culture really plays an important factor in this regard. Some learners, particularly those coming from an East Asian setting or a Southeastern one, tend to come from very conservative backgrounds. These learners will most likely feel intimidated talking to foreigners even in the educational setting. This intimidation is the consequence of the "face-saving" concept, which these learners believe in. If the reviewer has come from a different cultural background than the reviewee's, his/her feedback might not be really adopted since the reviewee views him/her as a foreigner, and the concept of "avoid foreigners" will most likely be "resurrected" (Monfret 1). These learners might prefer to isolate themselves and become more introvert if they feel alienated. This tends to happen when they are in an environment where they interact with foreigners.

The second factor that might impact the ways in which diverse groups of students can be scaffolded is the-same-age-group factor. Some learners-coming from East Asia and Southeastern countries - tend not to fully embrace feedback unless the source of such feedback is an authority figure - say a teacher. This does not mean that they do not welcome their trained-peers' feedback; it just means that the amount of feedback given by the trained peer might not be wholly addressed, incorporated, and adopted by the reviewee (Sultana 12). According to Sultana, these students see the teacher's feedback as an important role that the teacher should play in a classroom. They even view such feedback as the most significant.

Based on my experience in working with international students, especially some of those who have come from the aforementioned cultural backgrounds, it all has to do with age-influenced-by-pride factors. That is, by adopting my peer feedback, I am acknowledging that (s) he is academically superior to me. So, the concept of "academic inferiority and superiority" becomes active here, which might render the approach of training student reviewers, as a form of scaffolding, falling short to yield the desired outcomes.

Maybe by teaching students (reviewers and reviewees) how to be flexible and adaptable, attending to the surrounding milieus, we could help them learn to negotiate meaning, context, and audience. Students might benefit if teachers strive to instill in them the notion of making concessions based on logicality and rationality.

The third factor that might impact the ways in which diverse groups of students can be scaffolded is that of the participants' bias and pride. This is actually the very notion that $\mathrm{Lu}$ in her article "Professing Multiculturalism: The Politics of Style in the Contact Zone" asserts. She touches on the biases that interlocutors might display in rhetorical situation. She talks about how perpetuating the stereotype of the outsiders' and experts' compositions can play a major role in the manner that we approach each of these categories' compositions. She attributes this stereotype to the historical power that forms the base for this division. She cites Gertrude Stein's story with the "very nice American young man," whose press (Grafton Press) has sent him to check on Stein when she has made some arrangements to have her book Three Lives printed by Grafton. The story informs the reader of the criteria that "educated America" and, maybe, other cultures use to deal with a style of a particular idiosyncrasy. Two criteria are used: the writer's knowledge of English, which predicates on whether the writer is a native speaker of English or not; and the writer's experience in composition, which predicates on the notion whether s (he) has been "[im] perfectly educated" (Lu 444). According to Lu, the two major denominators whether a particular style representing "innovative experimentation or blundering "errors"' are ethnic and educational backgrounds (444).

\subsection{Peer Review Methodology: The Significance of Rahimi's "Is Training Student Reviewers Worth its While?"}

In his article "Is Training Student Reviewers Worth its While? A Study of How Training Influences the Quality of Students' Feedback and Writing", Rahimi cites other scholars who have praised the significance of scaffolding - in writing - in general and peer feedback in particular as a positive factor conducive to the learner's development. He cites Villamil and De Guerrero:

Part of the great appeal of feedback is also derived, in our view, from its strong foothold in theoretical principles relating social interaction and mediation to individual development. These principles are best expressed in the sociocultural theory of the development psychologist Lev Vygotsky (68). 
Reflecting upon the notion of mediation as a scaffolding-based means, Rahimi cites Wertsch. He argues that students should be first "other regulated." This means that they should seek to reach their ZPD through the help and support of a more knowledgeable peer, and this can only happen through interaction. When these supported-by-an-expert-peer students reach their ZPD, they will be in a position to become independent and/or "self-regulated" (68).

Rahimi, who also cites Berg, offers important findings based on the case study he has conducted in this regard at Shiraz University in Iran. He claims that the significance of trained peers' feedback is immeasurable. He asserts that the significance is immeasurable not only on enhancing the quality of the reviewees' writings - both in terms of the formal aspects (grammar and punctuation) and/or the global ones (cohesion and development) - but also on the quality of the writings that the reviewers themselves produce on the aforementioned levels (87). He claims that students who receive some training tend to produce better well-developed compositions, better well-organized paragraphs, and better grammatically-structured compositions; and so do their reviewees, whose compositions are reviewed and commented on by those reviewers.

Rahimi's findings are, however, limited in the following ways: a pedagogical setting with a diverse body of students is absent, and the t-test results have shown differences in the participants' academic writing skills. In other words, to have avoided critics questioning the findings of a case study of this sort, Rahimi's study should have controlled a major variable: the study should have taken place in a diverse pedagogical setting. Rahimi's findings are also limited in terms of the results of the independent t-test. Rahimi has jumped to the conclusion that such a technique is very fruitful although the results of the independent t-test in regard to the participants' scores on the TOEFL, which has been given to participants to measure their English proficiency level, distort and jab at his findings and conclusions. As far as the t-test results of his study are concerned, the probability value is greater than $0.05(\mathrm{t}=2.55, \mathrm{p}>0.05)$, which means that the proficiency level of the two groups is not equal (72). This factor alone tends to trigger us to question Rahimi's findings.

\section{Methodology}

\subsection{Intervention}

My planned intervention to address training student reviewers to provide feedback to one another, as well as to address how successful the means or tool used to achieve this purpose, includes the followings:

1. Lessons addressing both the global and formal levels of the writing process as some of the fundamental objectives of WRTG 1150 (First-Year Writing and Rhetoric)

2. Handouts with practicing exercises tackling both levels of the writing process for almost every major assignment required in the course

3. Dr. Andrea and Jennifer's peer-review letter

\subsection{Data Collection}

The data collection tools I have used are the followings:

1. Direct in-class observation of the students' interaction and collaboration during the peer-review process

2. Students' feedback and responses to their peers on the handout and the practicing exercises

3. Students' interviews and their reflections on the peer-review process

4. Students' feedback and responses to their peers in the light of the "tweaked" questions in Dr. Andrea and Jennifer's letter

It should be pointed out that my major focus was four students. Three are native speakers of Chinese and have come from a Chinese cultural background, and one is a native speaker of Saudi Arabian Arabic and has come from a Saudi Arabian cultural background. The reason for narrowing down the number of my focus group to four students is, involving all my class as the target group in this study, which is and will be part of a large-scale one that will go beyond the objectives of WRTG 1150 in terms of incorporating code-switching and code-meshing in the peer-review process, tends to take a longer time of organization and planning, which the time frame of the course I am teaching does not 
serve. Also, more students taking part in these peer-review processes requires investing more lectures in this process, which can only be done at the expense of overlooking other course objectives, let alone going beyond the main objectives of this class. That is said, this case study is only a small picture of a large-scale one, which will incorporate both code-switching and code-meshing and which I am planning to conduct over the summer in collaboration with the Department of Foreign Languages at Xi'an Jiaotong University in Xi'an, China.

\subsection{The Peer-Review Process Phases}

It should be pointed out that this case study wasset to have been designed as follows:

The process was supposed to take placein two phases over the course period. Each participant changed his/her partners at my discretion. Henceforth, I will refer to the participants involved in this case study by assigning each one of them a letter to distinguish him/her from other participants Student A, Student B, Student C, and Student D (Student D is the one from Saudi Arabia with Arabic background). In the first-peer review process, Student A worked with Student B while Student C worked with Student D. In the second peer review process, Student A worked with Student D while Student B worked with Student C. The same scenario was adopted for the peer-review-letter phase. Due to the lack of collaboration from two of the participants in the second phase of the peer-review (the peer-review letter), I had to revamp my methodology as follows:

\subsubsection{Phase One}

During this phase of the peer-review process, I have divided my students into pairs. Each student was given a handout-out with a list of questions addressing both the formal level and the global level of the writing process. Students then had to exchange their drafts with their peers. Each student had to read his/her peer's draft, read the questions on the handout, and answer them while providing feedback to his/her peer for the purpose of having his/her peer revise and come up with a better revised version of his/her rhetoric. Copies of these handouts are attached to this report in Appendix A and Appendix B. In other words, each pair played the role of a reviewer and reviewee to each other. In this phase, student A worked with Student B while student C worked with Student D.

\subsubsection{Phase Two}

I have used Dr. Andrea and Jennifer'speer-review letter. The students had to provide feedback to their peers by having attended to some questions that had showed up in the letter. The students had to provide feedback to their peers in the form of a formal letter, using email correspondence and carbon copying their teacher during this process. The students then had to respond to each other-also carbon copying me- (dis) incorporating their peer's feedback while having provided justification for whatever approach they had adopted. In consistency with Phase one, Student A worked with Student B while Student C worked with Student D. Copies of the peer-review letter are attached to this report in Appendix C, D.

Upon having completed both phases, the students' feedback and comments were analyzed. This analysis took more of a quantitative counting approach. That is, the number of comments incorporated on both levels of the writing process (global and formal) during each phase were calculated and compared for better understanding of the most effective technique having been harnessed to achieve the peer-review process. This approach was also used to obtain a better picture of the advantages versus the disadvantages/obstacles of this technique in general.

\subsubsection{Phase Three}

The students involved in this case study were interviewed to elicit their responses about the peerreview process they had participated in, the feedback they had exchanged, and the advantages/disadvantages of each phase they had taken part in.

\section{Data Analysis}

\subsection{Phase One}

\subsubsection{Student A with Student B}

Student A has provided two comments to his partner on the global level of his partner's rhetoric, more specifically in regard to his partner's thesis statement, which his partner seems to have incorporated in 
his revised work. No other comments have been given on the global level, as well as the formal one, of the writing process although Question B on Handout 1 and Question E on Handout have asked for the strengths and weaknesses of the draft on both levels.

Student B, on the other hand, has suggested a thesis statement, albeit a short one. He has also come up with three comments targeting the global level of the writing process, which his partner has seemed to be willing to incorporate into the revised version of his work. What is striking here is that both of these students, who have come from the same linguistic and cultural backgrounds, i.e., Chinese, have not provided any comment or feedback to each other on the formal level of the writing process (see table 1).

Table1. Number of comments given by Student A and Student B to each other and the number of comments agreed upon for incorporation in the revised work during the in-class-peer-review process

\begin{tabular}{|l|l|l|l|l|}
\hline Student & $\begin{array}{l}\text { Number of comments } \\
\text { (global level) }\end{array}$ & $\begin{array}{l}\text { Number of comments } \\
\text { (formal level) }\end{array}$ & $\begin{array}{l}\text { Total number of } \\
\text { comments }\end{array}$ & $\begin{array}{l}\text { Comments } \\
\text { incorporated }\end{array}$ \\
\hline A & 2 & 0 & 2 & 2 \\
\hline B & 4 & 0 & 4 & 4 \\
\hline
\end{tabular}

\subsubsection{Student $C$ with Student $D$}

Student $C$ has provided three comments to her partner in regard to his thesis statement, which Student $\mathrm{D}$ has agreed upon. However, she has only provided two comments in regard Question E on Handout 2 , the one asking about the global and the formal level of the writing process. These two comments have targeted the global level of the writing process. That is, she has not provided any feedback to her partner on the formal level of the writing process although Question B on Handout 1 and Question E on Handout have asked for the strengths and weaknesses of the draft on both levels.

Student D, on the other hand, has provided four comments to his partner in regard to her thesis statement, which his partner has agreed upon. He has not, however, provided any feedback in regard to Question B on Handout 1 and Question E on handout 2, which target the other global aspects of the writing process, as well as its formal ones (see table 2).

Table2. Number of comments given by Student $C$ and Student $D$ to each other and the number of comments agreed upon for incorporation in the revised work during the in-class-peer-review process

\begin{tabular}{|l|l|l|l|l|}
\hline Student & $\begin{array}{l}\text { Number of comments } \\
\text { (global level) }\end{array}$ & $\begin{array}{l}\text { Number of comments } \\
\text { (formal level) }\end{array}$ & $\begin{array}{l}\text { Total number of } \\
\text { comments }\end{array}$ & $\begin{array}{l}\text { Comments } \\
\text { incorporated }\end{array}$ \\
\hline C & 5 & 0 & 5 & 5 \\
\hline D & 4 & 0 & 4 & 4 \\
\hline
\end{tabular}

\subsection{Phase Two}

\subsubsection{Student A with Student B}

The peer-review letter has been introduced and used in this stage. Although students have been asked to explore the questions in the peer-review letter in class, and although they have seemed to be writing down some notes, neither one has provided any feedback to his peer through e-mail, which is the fundamental tool required for communicating and providing feedback in this phase. It should be pointed out here that both students are Chinese freshmen (see table 3).

Table3. Number of comments given by Student A and Student B to each other and the number of comments agreed upon for incorporation in the revised work during the peer-review-letter process

\begin{tabular}{|l|l|l|l|l|}
\hline Student & $\begin{array}{l}\text { Number of comments } \\
\text { (global level) }\end{array}$ & $\begin{array}{l}\text { Number of comments } \\
\text { (formal level) }\end{array}$ & $\begin{array}{l}\text { Total number of } \\
\text { comments }\end{array}$ & $\begin{array}{l}\text { Comments } \\
\text { incorporated }\end{array}$ \\
\hline A & 0 & 0 & 0 & 0 \\
\hline B & 0 & 0 & 0 & 0 \\
\hline
\end{tabular}

\subsubsection{Student $C$ with Student $D$}

Student C has provided three comments to Student D on the global level of the writing process. These comments have targeted the thesis statement and the development of Student D's argument. However, student D has agreed to adopt only one comment, which has targeted the conclusion of his argument. One comment regarding the thesis statement student D has rejected, arguing that it lacks a clear warrant. He has also rejected the third comment, which has targeted the development of his argument, 
arguing that it is very abstruse and lacks clarity. No comments on the formal level of the writing process have been suggested, and the answers to the questions addressing this level have been left blank.

Student D, on the other hand, has provided seven comments to Student C. All of these comments have targeted the global level of the writing process in Student C's draft. Three comments have targeted the thesis statement, and four comments have targeted the development of ideas in the draft. Student $\mathrm{C}$, however, has agreed to adopt four comments only: two addressing the thesis statement and two addressing the development of the argument in general. The other three comments have been rejected, which Student $\mathrm{C}$ has argued that they tend to make her argument two wordy and cumbersome to the reader. No comments have been suggested on the formal level of the writing process, and the answers to the questions addressing this level have been left blank (see table 4).

Table4. Number of comments given by Student $C$ and Student $D$ to each other and the number of comments agreed upon for incorporation in the revised work during the peer-review-letter process

\begin{tabular}{|l|l|l|l|l|}
\hline Student & $\begin{array}{l}\text { Number of comments } \\
\text { (global level) }\end{array}$ & $\begin{array}{l}\text { Number of comments } \\
\text { (formal level) }\end{array}$ & $\begin{array}{l}\text { Total number of } \\
\text { comments }\end{array}$ & $\begin{array}{l}\text { Comments } \\
\text { incorporated }\end{array}$ \\
\hline C & 3 & 0 & 3 & 1 \\
\hline D & 7 & 0 & 7 & 4 \\
\hline
\end{tabular}

Based on the results obtained, it seems to me that while the traditional way of the peer-review process has worked for all students, it has been more useful to Student A and Student B than the peer-review letter. I assume that direct observation of students through in-class interaction during the peer-review process seems to be helpful to different age categories than others. Student A and Student B are both freshmen, and it might be the case that the direct observation of their interaction in class has put some pressure on them to be more productive in terms of the peer-review process. However, when these students are not observed face-to-face in the peer-review letter, the task has not been taken seriously, which has impacted their performance by falling short to provide any feedback or comments to each other. I assume that in-class observation might be efficient for some age categories than others. The same holds true for the peer-review letter.

On other hand, it seems to me that the peer-review letter has been a good pedagogical tool that has worked well for Student C and Student D, who are in more advanced academic levels than Student A and Student B. When I compare the amount of feedback given by these students to each other during each phase of the peer-review process, I realize that these students have been more productive during the second phase of the peer-review process, the peer-review-letter phase. Also, although these students have provided almost the same amount of feedback during both phases of the peer-review process, their feedback during the in-class-peer-review process, to a certain extent, can be described as perfunctory. However, their feedback has been insightful and thorough during the peer-reviewletter process. I assume that the time factor has played a very major role in the in-class-peer-review process. That is, maybe due to the class time limitation, these students have not had enough time to delve deep into each other's draft and provide insightful feedback to one another. However, when the time factor has not been an obstacle in the peer-review letter process, they have delved into each other's draft and provided more insightful and thorough feedback. Also, when compared with Student A and Student B, I assume that Student C and Student D's academic levels have played a very major role in their performance, being responsible and productive, an aspect that Student A and Student B have lacked in this stage.

\subsection{Phase Three}

\subsubsection{Interviews}

Although I have attempted to interview all of my participants, only Student B, Student C, and Student D have taken part in the interview as Student A has backed out of it. This again makes me question the role that the participants' ages have played in this case-study. The feedback that I have obtained from Student C and Student D is that the peer-review process has been helpful to them in terms of helping them find out their writing weaknesses and strengths and try to work on the weak aspects. They have also indicated that the peer review process has helped them look at the topic they are tackling from different angles as their peers have always raised interesting points thy have never thought of, particularly on the global level of the writing process. They have reported that the peer- 
review process provides a mutual benefit for both parties. When the students have been asked to reflect on which pedagogy of the peer- review process they like most or find more helpful/useful, they have indicated that the traditional approach is better and more helpful as this approach helps them discuss the exchanged feedback face-to-face as some of the feedback they have exchanged through email lacks elaboration. Students have also indicated that their grammar is not good, which has seemed one of the reasons that has refrained them from providing some feedback on the formal level of the writing process. Student B, on the other hand, has reported that he thinks that the peer-review process is helpful. However, he has reported that he is inclined to work with other students from the same linguistic background, i.e., Chinese. He has indicated that he does not feel comfortable working with students from other linguistic backgrounds. His answer has reinforced Monfret's notion of the "face-saving concept."

\section{DisCUSSION}

The purpose of my action research project is to examine the benefit of peer-review in writing class as a form of scaffolding, as well as the best pedagogical tool that can be adopted by the teacher to achieve this process.

My results tell me that the peer-review process is generally helpful for both the reviewer and the reviewee. They also tell me that there is no winner-takes-all pedagogical tool that can be efficient for all students, regardless of their ages and academic levels. That is, different pedagogical tools can be helpful based on the surrounding milieu. The teacher has to adopt the appropriate tool, attending to the age and the academic level of his students. While direct observation of students seems to work well with younger students whose academic levels are less advanced, it does not seem so with older students whose academic levels are more advanced. As the results tell me here, older and more responsible students have done better in terms of providing feedback to their peers in the peer-review letter process than in the in-class peer-review one. On the contrary, younger students have performed well on the latter than the former.

The results I have obtained from this action research project have piqued my interest to conduct a different case study to see if my initial reading and interpretation of the results I have obtained are true. My inquiries for such case study would be:

1. Should the teacher use the peer-review letter with older, more academically advanced students while the traditional peer-review process with younger, less academically advanced ones?

2. Why do not students seem to provide feedback to their peers on the formal level of the writing process? Could it be the case that they have developed a sort of phobia on this level, attending to the fact that they are not native speakers of the language in question?

3. Why do students tend to reject some of their peers' feedback in the peer-review letter process, which is not carried out face-to-face, while not doing so in the face-to-face peer-review process? Could it be the case that students consider saving their peers' faces during the face-to-face peerreview processes?

\section{REFERENCES}

Canagarajah, Suresh. "Codemeshing in Academic Writing: Identifying Teachable Strategies of Translanguaging." The Modern Language Journal 95.iii (2011): 401-417. Print.

"Toward a Writing Pedagogy of Shuttling between Languages: Learning from Multilingual Writers." College English 68.6 (July 2006): 589-604. Print.

$\mathrm{Lu}$, Min-Zhan."Professing Multiculturalism: The Politics of Style in the Contact Zone."College Composition and Communication (CCC) 45.4 (December 1994): 442-458. Print.

Monfret, Anne-Laure."Saving and Losing Face.”Culture (2011): 12-13. Web. 21 Dec., 2015.

Rahimi, Mohammad. "Is Training Student Reviewers Worth its While? A Study of How Training Influences the Quality of Students' Feedback and Writing." Language Teaching Research 17.1 (2013): 67-89. Print.

Shoebottom, Paul. "ESL Workshop: Scaffolding Theory." ESL Workshop: Scaffolding Theory. 2015. Web. 20 Sept. 2015.

Sultana, Asifa."Peer Correction in ESL Classrooms." BRAC University Journal V1.1 (2009): 11-19.Web. 24 Dec., 2015. 


\section{AUTHOR'S BIOGRAPHY}

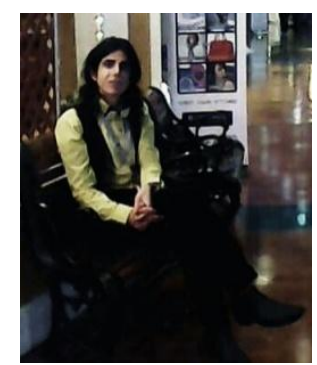

Jamal A. Khlifat, works in the field of Linguistics at University of Colorado, Boulder United States. His research interests are forensic linguistics, phonetics, phonology, documentation of the world's endangered languages, pharyngealization and pharyngeal harmony in Levantine Arabic, gemination in Levantine Arabic, nasalization and nasal geminates in Levantine Arabic, syntax, lexical semantics, prosody, and rhetoric and Toulmin argumentation.

He was born with a malleus in his ear enabling him to distinguish the different sounds exist in the disputed number of the world's languages. He is into anatomizing those abstract units called phonemes to examine the phonetic variations that can be observed. There is nothing as intriguing as tracing the different forms a spoken message exists in its progress from the speaker's mind to the listener's. How this peculiar linguistic signal is converted into an acoustic one, which is, in turn, converted into an electro-chemical signal in the brain, to be later on decoded into segments; words; and, ultimately, meaning, reveals the supernatural power that has created this physiological linguistic apparatus. 